\title{
Scalar Tensor Teleparallel Dark Gravity via Noether Symmetry
}

\author{
Yusuf Kucukakca* \\ Department of Physics, Faculty of Science, Akdeniz University, 07058 Antalya, Turkey
}

(Dated: March 8, 2018)

\begin{abstract}
In this paper, in the framework of teleparallel gravity we consider scalar tensor theories of gravity in which scalar fields are nonminimal coupled to torsion scalar. Noether symmetry of the Lagrangian of such a theory for the Friedman-Robertson-Walker spacetime is used to determine the explicit forms for the coupling function and for the potential, and it is shown that both must be power-law forms as a functions of the scalar field. The solutions of the field equations for the considered models are presented by using the results obtained from the Noether symmetry. It is shown that the equation of state parameter in the present model can cross the phantom divide line for a special case with the coupling function $F(\phi)=\frac{3}{16} \phi^{2}$ and for the potential $V(\phi)=\lambda \phi^{2}$.

PACS numbers: 04.50.+h, 04.40.Dg, 97.60.Gb
\end{abstract}

\section{INTRODUCTION}

In the past decade there is a general consensus that today our universe is undergoing an accelerated expansion. This result with the accelerated expansion becomes the central theme of the modern cosmology and confirms various observational evidences which are the observations of supernovae Type Ia (SNe Ia) [1 -3], cosmic microwave background radiation $(\mathrm{CMB})$ [4, 5], and large-scale structure [6]. The standard cosmology can not clarify what causes this cosmic acceleration and cosmologists have to seek a powerful explanation for this observed realty in observational cosmology. In order to explain the accelerated behaviour of the universe in framework of General Relativity (GR) one can produce a dark fluid with a negative pressure which named the dark energy. A simple candidate of dark energy is the cosmological constant with the equation of state (EoS) parameter, $w=-1$. However, the cosmological constant model is subject to the so-called fine-tuning and coincidence problems [7, 8]. To solve these problems, various dynamical dark energy models have been proposed. The simplest one is a canonical scalar field which is dubbed quintessence dark energy that has been extensively studied in the literature $9-12$. Another one is to work a scalar field with a negative kinetic energy, dubbed phantom dark energy [13 16]. Finally, quintom scenario combining the quintessence and phantom dark energy has been proposed [17-19]. In such a model EoS parameter can pass below and up the phantom divide line $w=-1$.

On the other hand alternative approach dealing with the acceleration problem of the universe is to modified the geometric part of Einstein field equation. Such a modifications in GR is known as $f(R)$ gravity theory. The simplest form of this theory can be constructed by replacing the Ricci scalar $R$ with an arbitrary function $f(R)$ in the EinsteinHilbert Lagrangian (for reviews see e.g. 20-23]).

It is known that teleparallel equivalent of GR, referred to as teleparallel gravity, was firstly introduced by Einstein to unify the gravity and the electromagnetism [24, 25]. In this gravity theory, the equations of motion for any spacetime are exactly the same as of GR, but, instead of using the curvature defined by the Levi-Civita connection, it uses the Weitzenböck connection that has no curvature but only torsion. Similar to GR, where the action is a curvature scalar, the action of teleparallel gravity is a torsion scalar $T$. The dynamical variable of the teleparallel gravity is the vierbein fields and the field equations are obtained by taking variation of the action with respect to the vierbein fields [26]. Some modifications in the teleparallel gravity theory have been recently proposed in order to get a solution for the cosmic acceleration of the universe without introducing the dark energy. A particular class of modified gravity theories is the $f(T)$ gravity theory which can be constructed by replacing the torsion scalar $T$ with an arbitrary function $f(T)$ in the action of the teleparallel gravity 27 30]. We note that the field equations in this theory are second-order differential equations, while for the generalized $f(R)$ theory they are fourth-order. Lately, $f(T)$ theory attracted much attention in the literature, and we refer to e.g. 31 48] for some relevant works.

Very recently, new scenario the so-called teleparallel dark energy has been proposed by Geng et al in which they added the quintessence scalar field non-minimally coupled to the torsion scalar in the framework of teleparallel gravity 495 53. They have found that such a theory has a richer structure than the same one in the framework of general relativity. The richer structure of non-minimally coupled scalar field with the torsion scalar is due to exhibiting

*Electronic address: ykucukakca@akdeniz.edu.tr 
quintessence-like or phantom-like behavior, or experiencing the phantom divide crossing in this theory. We note that in the minimal coupling case, cosmological model with the quintessence scalar field in teleparallel gravity is identical to that in GR. Motivated by the teleparallel dark energy scenario, we here extend it by replacing the coupling function term $1+\xi \phi^{2}$ where $\xi$ is a non-minimal coupling constant, with the arbitrary function $F(\phi)$. In such a scenario it is important to determine the form of $F(\phi)$. Noether symmetry approach that was introduced by Capozziello et al., allows one to choose the potential and the coupling function dynamically in the scalar-tensor gravity theory. Utilizing this approach we find the potential and the coupling function in the teleparallel dark energy scenario and we solve the analytically the field equations of the theory. Our results show that the teleparallel dark energy equation of state parameter has quintessence, phantom-like behavior or the transition from quintessence to phantom phase in this theory. In particular, when we choose the quadratic potential and coupling function via Noether symmetry approach, we obtain that during the evolution of the universe the equation of state parameter changes from $w_{\phi}>-1$ to $w_{\phi}<-1$.

This paper is organized as follows. In the following section, the field equations are derived from a point-like Lagrangian in a Friedman-Robertson-Walker spacetime, which is obtained from an action ingluding the scalar field non-minimally coupled to the torsion scalar in the framework of teleparallel gravity. In the Section III we search the Noether symmetry of the Lagrangian of scalar tensor theory and give the solutions of the field equations by using Noether symmetry approach. Finally, in the Section IV] we conclude with a brief summary of the obtained results.

\section{TELEPARALLEL GRAVITY WITH SCALAR FIELD}

Let us consider the general action for a scalar field, non minimally coupled with the torsion scalar, in this case we write

$$
S=\int d^{4} x e\left[F(\phi) T+\frac{1}{2} \partial_{\mu} \phi \partial^{\mu} \phi-V(\phi)\right]
$$

where $e=\operatorname{det}\left(e_{\mu}^{i}\right)=\sqrt{-g}$ that $e_{\mu}^{i}$ is tetrad (vierbein) basis, $T$ is a Torsion scalar, $F(\phi)$ is the generic function that describe the coupling and $V(\phi)$ is the potential for the scalar field. Note that we use Planck units. For $F(\phi)=1+\xi \phi^{2}$, the action (11) reduce to the action which proposed by Geng et al [49]. We also note that the action (11) with the torsion formulation of GR is similar to the standard scalar tensor theory where the scalar field couples to the Ricci scalar [54].

The homogeneous and isotropic Friedmann-Robertson-Walker universe is described by the metric

$$
d s^{2}=d t^{2}-a^{2}(t)\left(d x^{2}+d y^{2}+d z^{2}\right)
$$

where $\mathrm{a}(\mathrm{t})$ is the scale factor. It has been found in Ref. [55] that the torsion scalar in the teleparallel gravity can be expressed as $T=-\frac{6 \dot{a}^{2}}{a^{2}}$. Considering the background in (2), it is possible to obtain a point-like Lagrangian from action (11)

$$
L=-6 F(\phi) a \dot{a}^{2}+a^{3}\left(\frac{\dot{\phi}^{2}}{2}-V(\phi)\right),
$$

here, a dot indicates differentiation with respect to the cosmic time $t$. It is well known that the Euler-Lagrange equations for a dynamical system are

$$
\frac{\partial L}{\partial q_{i}}-\frac{d}{d t}\left(\frac{\partial L}{\partial \dot{q}_{i}}\right)=0
$$

where $q_{i}$ are the generalized coordinates of the configuration space. The configuration space of the Lagrangian (3) is $Q=(a, \phi)$ and whose tangent space is $T Q=(a, \phi, \dot{a}, \dot{\phi})$. Substituting Eq. (3) into the Euler-Lagrange equation (44) for the scalar field, we obtain

$$
\ddot{\phi}+3 H \dot{\phi}+6 F^{\prime} H^{2}+V^{\prime}(\phi)=0 .
$$

which is a KleinGordon equation for the coupled scalar field. From the Euler-Lagrange equation for the scale factor $a$ by using the Lagrangian (3), we obtain the acceleration equation, namely

$$
2 \dot{H}+3 H^{2}=-\frac{p_{\phi}}{2 F} .
$$


Furthermore, the energy function (Hamiltonian) associated with the Lagrangian (3) is found as

$$
H^{2}=\frac{\rho_{\phi}}{6 F}
$$

which can be considered a constraint equation. In the above last equations, we define the energy density $\rho_{\phi}$ and the pressure $p_{\phi}$ of the scalar field as follows

$$
\begin{gathered}
\rho_{\phi}=\frac{1}{2} \dot{\phi}^{2}+V(\phi), \\
p_{\phi}=\frac{1}{2} \dot{\phi}^{2}-V(\phi)+4 F^{\prime} H \dot{\phi} .
\end{gathered}
$$

In order to solve the field equations, one has to choose a form for the coupling function and the potential density. To do this, in the following section we will use the Noether symmetry approach.

\section{NOETHER SYMMETRY APPROACH AND COSMOLOGICAL SOLUTIONS}

As is well known, the Noether theorem generates a conserved quantity in the classical mechanic. The application of this theorem to cosmology was introduced by De Rittis et al. [56, 57] and Cappoziello et al. [58, 59] to find preferred solutions of the field equations and the dynamical conserved quantity. The Noether theorem states that if the Lie derivative of a given Lagrangian $L$ dragging along a vector field $\mathbf{X}$ vanishes

$$
£_{\mathbf{X}} L=0 \text {. }
$$

then $\mathbf{X}$ is a symmetry for the dynamics, and it generates the conserved quantity. We also note that the Noether symmetry approach allows one to choose the potential dynamically in the scalar-tensor gravity theory [56 64, and the explicit form of the function $f(R)$ of the modified $f(R)$ theories of gravity 65 71]. In this form of $f(R)$, cosmological solutions in the case of FRW metric can describe the accelerated period of the Universe. Spherically symmetric solutions in $f(R)$ theories of gravity have been also found in [72], using the Noether symmetry approach. On the other hand, some authors specified the form of $f(T)$ in the modified $f(T)$ theories of gravity via Noether symmetry approach 73 76].

The existence of Noether symmetry implies the existence of a vector field $\mathbf{X}$ such that

$$
\mathbf{X}=\alpha \frac{\partial}{\partial a}+\beta \frac{\partial}{\partial \phi}+\dot{\alpha} \frac{\partial}{\partial \dot{a}}+\dot{\beta} \frac{\partial}{\partial \dot{\phi}}
$$

where $\alpha, \beta$ and $\gamma$ are depend on $a$ and $\phi$. Hence the condition given by (10) for the existence of a symmetry gives rise to the following set of coupled differential equations,

$$
\begin{aligned}
& \alpha+2 a \frac{\partial \alpha}{\partial a}+\frac{F^{\prime}}{F} a \beta=0 \\
& 3 \alpha+2 a \frac{\partial \beta}{\partial \phi}=0 \\
& 12 F \frac{\partial \alpha}{\partial \phi}-a^{2} \frac{\partial \beta}{\partial a}=0 \\
& 3 V \alpha+a V^{\prime} \beta=0
\end{aligned}
$$

This system are obtained by imposing the fact that the coefficients of $\dot{a}^{2}, \dot{a} \dot{\phi}$ and $\dot{\phi}^{2}$ vanish. Using the separation of variable one can find the solutions of the above set of differential equations (12)-(15) for $\alpha, \beta$, coupling function $F(\phi)$ and potential $U(\phi)$ as

$$
\begin{gathered}
\alpha=-\frac{2 \alpha_{0}}{2 n+3} a^{n+1} \phi^{-\frac{2 n}{2 n+3}}, \quad \beta=\alpha_{0} a^{n} \phi^{\frac{3}{2 n+3}}, \\
F(\phi)=\frac{(2 n+3)^{2}}{48} \phi^{2},
\end{gathered}
$$




$$
V(\phi)=\lambda \phi^{\frac{6}{2 n+3}}
$$

where $\alpha_{0}, \lambda>0$ and $n$ are a constants and $n \neq-3 / 2$. If we consider the minimal coupling case i.e. $F(\phi)=1 / 2$, the above Noether equations (12)-(15) give an exponential potential. This case is completely equivalent to standard quintessence model which has been studied in 77, 78. We note that for the physically acceptable of our model, the most important requirement is $G_{e f f}>0$ (Here we define the effective gravitational constant as $G_{e f f}=\frac{1}{2 F}$ ). Since the coupling function always positive for all the values of $n$, this condition is provided.

In the present form the analytical solutions of the field equations given by (5)-(7) are not straightforward to calculate. In order to integrate our the dynamical system, we search for a cyclic variable associated with the Noether symmetry. If there exist such a symmetry, then one can always perform a change of variables $(a, \phi) \rightarrow(z, u)$ such that the Lagrangian (3) becomes cyclic in one of them. We will see below that the dynamical system involving the cyclic variable can be easily solved. Following this procedure given by Ref.[79], we can introduce the new variables as

$$
z=\frac{2 n+3}{4 n \alpha_{0}} a^{-n} \phi^{\frac{2 n}{2 n+3}}, \quad u=a \phi^{\frac{2}{2 n+3}},
$$

in which the variable $z$ is a cyclic and $n \neq 0$. Then the scale factor and the scalar field can be rewritten as

$$
\begin{gathered}
a=u^{\frac{1}{2}}\left(\frac{4 n \alpha_{0} z}{2 n+3}\right)^{-\frac{1}{2 n}}, \\
\phi=u^{\frac{2 n+3}{4}}\left(\frac{4 n \alpha_{0} z}{2 n+3}\right)^{\frac{2 n+3}{4 n}} .
\end{gathered}
$$

In term of these new variables, considering the coupling function (17) and potential (18), the Lagrangian (3) takes a manageable form

$$
L=(2 n+3) \alpha_{0} u^{n+2} \dot{u} \dot{z}-2 \lambda u^{3} .
$$

It is clear that the variable $z$ is cyclic for this Lagrangian. The Euler-Lagrange equations for $z$ and $u$ and the Hamiltonian constraint equation $\left(E_{L}=0\right)$ associated with this Lagrangian are, respectively,

$$
\begin{gathered}
\dot{u}-\frac{\epsilon_{0}}{(2 n+3) \alpha_{0}} u^{-(n+2)}=0, \\
\ddot{z}+\frac{6 \lambda}{(2 n+3) \alpha_{0}} u^{-n}=0, \\
\dot{u} \dot{z}+\frac{2 \lambda}{(2 n+3) \alpha_{0}} u^{1-n}=0,
\end{gathered}
$$

where $\epsilon_{0}$ is an integration constant related with the constant of motion. Then the solutions $z$ and $u$ are obtained as

$$
\begin{gathered}
u(t)=\left(b_{1} t+c_{1}\right)^{-\frac{m}{n+3}}, \\
z(t)=b_{2}\left(b_{1} t+c_{1}\right)^{\frac{n+6}{n+3}}+c_{2},
\end{gathered}
$$

where $c_{1}$ and $c_{2}$ are an integration constants and we also define $b_{1}=-\frac{\epsilon_{0}(n+3)}{m \alpha_{0}(2 n+3)}$ and $b_{2}=-\frac{2 \lambda \alpha_{0} m^{2}(2 n+3)}{\epsilon_{0}^{2}(n+6)}, n \neq-6$. Putting the above expressions into Eq. (20), we find the general solutions for the scale factor and scalar field

$$
\begin{aligned}
& a(t)=\left(\frac{4 \alpha_{0} b_{2}}{2 n+3}\right)^{-\frac{1}{2 n}}\left(b_{1} t+c_{1}\right)^{-\frac{3}{n(n+3)}}, \\
& \phi(t)=\left(\frac{4 \alpha_{0} b_{2}}{2 n+3}\right)^{\frac{2 n+3}{4 n}}\left(b_{1} t+c_{1}\right)^{\frac{2 n+3}{2 n}},
\end{aligned}
$$

here we assume that $c_{2}$ is zero without loss of generality. The deceleration parameter, which is an important quantity in cosmology, is defined by $q=-a \ddot{a} / \dot{a}^{2}$, where the positive sign of $q$ indicates the standard decelerating 
models whereas the negative sign corresponds to accelerating models and $q=0$ corresponds to expansion with constant velocity. It takes the following form in this model

$$
q=-\frac{1}{3}\left(n^{2}+3 n+3\right)
$$

From Eq. (29) we see that the universe is accelerating for $n \in$ reel. We can also define the equation of state parameter for the scalar field by using Eqs. (6)-(9) as $w_{\phi} \equiv \frac{P_{\phi}}{\rho_{\phi}}=\frac{2 q-1}{3}$. Then it can be obtained by

$$
w_{\phi}=-\frac{1}{9}\left(2 n^{2}+6 n+9\right) .
$$

Astrophysical data indicate that $w$ lies in a very narrow strip close to $w=-1$. The case $w=-1$ corresponds to the cosmological constant. For $w<-1$ the phantom phase is observed, and for $-1<w<-1 / 3$ the phase is described by quintessence. Thus, in the intervals $-\infty<n<-3$ and $0<n<\infty$ we have phantom phase. If $-3<n<0$, then the quintessence phase occurs, where the universe is both expanding and accelerating.

Now, from the above solution we concentrate on the case $n=0,-3$ which provide, as will be shown shortly, an interesting class of models. Firstly we consider the case $n=0$.

\section{A. Case $n=0$}

For $n=0$, from Eqs. (17) and (18) the coupling function and the potential of scalar field result $F(\phi)=\frac{3}{16} \phi^{2}$ and $V(\phi)=\lambda \phi^{2}$.

With the same procedure as the above section we find the new transformations

$$
z=-\frac{3}{2 \alpha_{0}} \ln (a), \quad u=a^{\frac{3}{2}} \phi,
$$

and the inverse transformation

$$
a=e^{-\frac{2 \alpha_{0}}{3} z}, \quad \phi=u e^{\alpha_{0} z}
$$

We rewrite Eq. (3) using the inverse transformation Eq. (20) and the solutions for the coupling function $f(\phi)$ the potential $V(\phi)$ as obtained in the this section in the following form

$$
L=\alpha_{0} u \dot{u} \dot{z}+\frac{1}{2} \dot{u}^{2}-\lambda u^{2} .
$$

This Lagrangian yields following the equations of motion

$$
\begin{gathered}
\dot{u}-\frac{\epsilon_{1}}{\alpha_{0}} u^{-1}=0, \\
\ddot{u}+\alpha_{0} u \ddot{z}+2 \lambda u=0, \\
\frac{1}{2} \dot{u}^{2}+\alpha_{0} u \dot{u} \dot{z}+\lambda u^{2}+=0,
\end{gathered}
$$

where $\epsilon_{1}$ is a constant of motion for this case. The above equations have a solutions as

$$
\begin{gathered}
z(t)=-\frac{\lambda t^{2}}{\alpha_{0}}-\frac{c_{3} \lambda t}{\epsilon_{1}}-\frac{\ln \left(2 \epsilon_{1} t+\alpha_{0} c_{3}\right)}{4 \alpha_{0}}+c_{4}, \\
u(t)=\frac{\sqrt{\alpha_{0}\left(2 \epsilon_{1} t+\alpha_{0} c_{3}\right)}}{\alpha_{0}},
\end{gathered}
$$

where $c_{3}$ and $c_{4}$ are an integration constants. After returning to the original variables we obtain the scale factor and the scalar field

$$
a(t)=\left(2 \epsilon_{1} t+\alpha_{0} c_{3}\right)^{\frac{1}{6}} e^{\left[\frac{2 \alpha_{0}}{3}\left(\frac{\lambda t^{2}}{\alpha_{0}}+\frac{c_{3} \lambda t}{\epsilon_{1}}-c_{4}\right)\right]},
$$




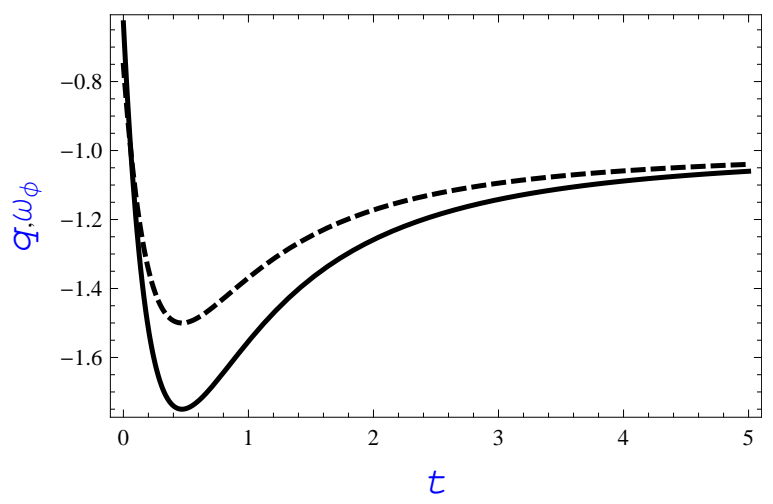

FIG. 1: For $n=0$, plots of the deceleration parameter (solid line) and the equation of state parameter (dashed line) with respect to cosmic time $t$. We take $\epsilon_{1}=\alpha_{0}=c_{3}=1$ and $\lambda=0.4$

$$
\phi(t)=\alpha_{0}^{-1 / 2}\left(2 \epsilon_{1} t+\alpha_{0} c_{3}\right)^{\frac{1}{4}} e^{\left[-\alpha_{0}\left(\frac{\lambda t^{2}}{\alpha_{0}}+\frac{c_{3} \lambda t}{\epsilon_{1}}-c_{4}\right)\right]} .
$$

Using the definition of the deceleration parameter and the equation of state parameter one can find

$$
\begin{gathered}
q=-1-\frac{6 \epsilon_{1}^{2}\left(8 \lambda \epsilon_{1}^{2} t^{2}+8 \lambda \epsilon_{1} \alpha_{0} c_{3} t+2 \lambda \alpha_{0}^{2} c_{3}^{2}-\epsilon_{1}^{2}\right)}{\left(8 \lambda \epsilon_{1}^{2} t^{2}+8 \lambda \epsilon_{1} \alpha_{0} c_{3} t+2 \lambda \alpha_{0}^{2} c_{3}^{2}+\epsilon_{1}^{2}\right)^{2}}, \\
w_{\phi}=-1-\frac{4 \epsilon_{1}^{2}\left(8 \lambda \epsilon_{1}^{2} t^{2}+8 \lambda \epsilon_{1} \alpha_{0} c_{3} t+2 \lambda \alpha_{0}^{2} c_{3}^{2}-\epsilon_{1}^{2}\right)}{\left(8 \lambda \epsilon_{1}^{2} t^{2}+8 \lambda \epsilon_{1} \alpha_{0} c_{3} t+2 \lambda \alpha_{0}^{2} c_{3}^{2}+\epsilon_{1}^{2}\right)^{2}} .
\end{gathered}
$$

In order to determine evolution of the equation of state parameter and the deceleration parameter, we present the results in Figure 1 This figure clearly shows that crossing of the phantom divide line $w_{\phi}=-1$ can be realized from the quintessence phase $w_{\phi}>-1$ to the phantom phase $w_{\phi}<-1$ in our model. It is interesting to note that such a crossing is agreement with the recent cosmological observational data 80 82].

\section{B. Case $n=-3$}

From Eqs. (17) and (18), the coupling function has the same form as the case $n=0$ but the potential of scalar field results $V(\phi)=\frac{\lambda}{\phi^{2}}$ which is the well-known inverse square potential and has been studied in detail [83 86$]$.

With the same procedure as the above section we find the new transformations

$$
z=\frac{a^{3} \phi^{2}}{4 \alpha_{0}}, \quad u=a^{-\frac{3}{2}} \phi
$$

Therefore the Lagrangian (3) takes the form

$$
L=\alpha_{0} \frac{\dot{u} \dot{z}}{u}-\frac{\lambda}{u^{2}}
$$

For this Lagrangian, the Euler-Lagrange equations and Hamiltonian constrained equation provide the following equations of motion

$$
\begin{gathered}
\dot{u}-\frac{\epsilon_{2}}{\alpha_{0}} u=0, \\
\ddot{z}-\frac{2 \lambda}{\alpha_{0}} u^{-2}=0, \\
\dot{u} \dot{z}+\frac{\lambda}{\alpha_{0}} u^{-1}=0,
\end{gathered}
$$




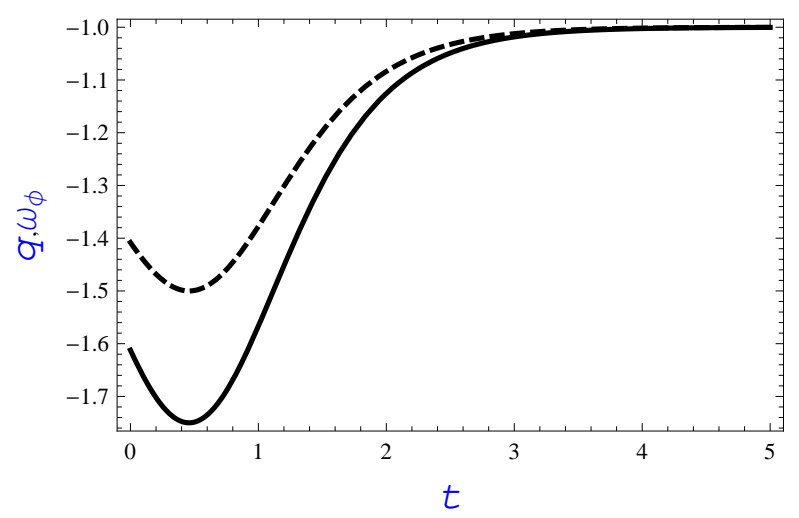

FIG. 2: For $n=-3$, plots of the deceleration parameter (solid line) and the equation of state parameter (dashed line) with respect to cosmic time $t$. we choose $\lambda=\alpha_{0}=c_{5}=c_{6}=1$ and $\epsilon_{2}=-1$.

where $\epsilon_{2}$ is a constant of motion for this case. The above equations have a solutions as

$$
z(t)=\frac{\lambda \alpha_{0}}{2 \epsilon_{2}^{2} c_{5}^{2}} e^{-\frac{2 \epsilon_{2} t}{\alpha_{0}}}+c_{6},
$$

and

$$
u(t)=c_{5} e^{\frac{\epsilon_{2} t}{\alpha_{0}}}
$$

where $c_{5}$ and $c_{6}$ are integration constants. After returning to the original variables we obtain the scale factor and the scalar field, respectively,

$$
a(t)=\left[\frac{2 \alpha_{0} e^{-\frac{2 \epsilon_{2} t}{\alpha_{0}}}}{\epsilon_{2}^{2} c_{5}^{4}}\left(\lambda \alpha_{0} e^{-\frac{2 \epsilon_{2} t}{\alpha_{0}}}+2 \epsilon_{2}^{2} c_{5}^{2} c_{6}\right)\right]^{1 / 6},
$$

and

$$
\phi(t)=\left[\frac{2 \alpha_{0} e^{\frac{2 \epsilon_{2} t}{\alpha_{0}}}}{\epsilon_{2}^{2}}\left(\lambda \alpha_{0} e^{-\frac{2 \epsilon_{2} t}{\alpha_{0}}}+2 \epsilon_{2}^{2} c_{5}^{2} c_{6}\right)\right]^{1 / 4} .
$$

In this case the deceleration and equation of state parameters become

$$
\begin{gathered}
q=-1-\frac{3 \lambda \alpha_{0} \epsilon_{2}^{2} c_{5}^{2} c_{6} e^{-\frac{2 \epsilon_{2} t}{\alpha_{0}}}}{\left(\lambda \alpha_{0} e^{-\frac{2 \epsilon_{2} t}{\alpha_{0}}}+\epsilon_{2}^{2} c_{5}^{2} c_{6}\right)^{2}}, \\
w_{\phi}=-1-\frac{2 \lambda \alpha_{0} \epsilon_{2}^{2} c_{5}^{2} c_{6} e^{-\frac{2 \epsilon_{2} t}{\alpha_{0}}}}{\left(\lambda \alpha_{0} e^{-\frac{2 \epsilon_{2} t}{\alpha_{0}}}+\epsilon_{2}^{2} c_{5}^{2} c_{6}\right)^{2}} .
\end{gathered}
$$

If we choose $c_{6}=0$, we obtain the de Sitter solution. In Figure 2 and Figure 3 behaviors of these parameters, by a suitable choice of the constants $b_{6}$ or $\alpha_{0}$, are depicted in terms of the cosmic time $t$. From these figures we have both the phantom $\left(b_{6}>0\right.$ or $\left.\alpha_{0}>0\right)$, and quintessence phase $\left(b_{6}<0\right.$ or $\left.\alpha_{0}<0\right)$. In this case, the phantom divide line is never crossed.

\section{CONCLUSIONS}

It has recently been proposed as an alternative dark energy scenario [49 53] and so-called teleparallel dark energy. In these literatures,authors have added a scalar field to the Teleparallel Equivalent to General Relativity, allowing for 


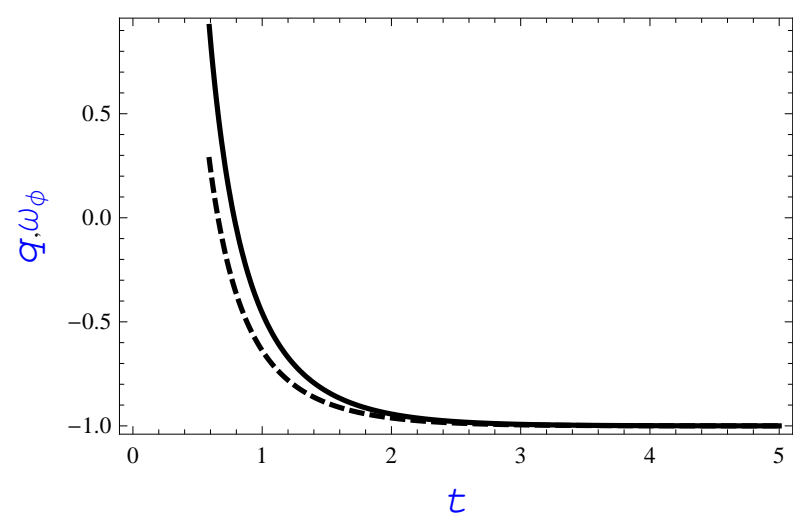

FIG. 3: For $n=-3$, plots of the deceleration parameter (solid line) and the equation of state parameter (dashed line) with respect to cosmic time $t$. we choose $\lambda=\alpha_{0}=c_{5}=1, c_{6}=-1$ and $\epsilon_{2}=-1$.

a non minimal coupling between the field and the torsion scalar. Motivated by this scenario, in the present work, we have investigated the scalar tensor dark energy models, where the gravitational part of the Lagrangian is $F(\phi) T$. Such a model may have a richer structure than the same one in the framework of GR. If we choose the coupling function as $F(\phi)=1+\xi \phi^{2}$, then our model reduced to Lagrangian which proposed by Geng et al [49]. As we said above, the Noether symmetry approach a very important tool, because it guarantees the conservation laws and restricts the possible expressions for the coupling function and for the potential of scalar field in the framework of teleparallel gravity. Further, this approach leads to a variable transformation that usually makes it possible to obtain exact and general solutions for the evolution of the scale factor and the scalar field. Here, using the Noether symmetry approach we construct the explicit forms of the coupling function and potential as $F(\phi)=\frac{(2 n+3)^{2}}{48} \phi^{2}$ and $V(\phi)=\lambda \phi^{\frac{6}{2 n+3}}$. Such a models yields a power law expansion for the cosmological scale factor (see Eq. (27)). We have also presented the equation of state parameter for our model. It has been turned out that a phantom like dark energy for the intervals $-\infty<n<-3$ and $0<n<\infty$ and a quintessence like dark energy for the interval $-3<n<0$ occur. In two special cases, for $n=0$, we have obtained the coupling function as $F(\phi)=\frac{3}{16} \phi^{2}$ and the potential as $V(\phi)=\lambda \phi^{2}$. For the model, we present the results in Fig. 1 where shows that crossing of the phantom divide line $w_{\phi}=-1$ can be realized from the quintessence phase $w_{\phi}>-1$ to the phantom phase $w_{\phi}<-1$. For $n=-3$, the coupling function has a same form but the potential has inverse square form. Our results for the equation of state parameter have depicted in Fig. 2 and Fig. 3. From these figures we observed that the phantom divide line is never crossed. Therefore, by considering the above results it seems that the quadratic potential $V(\phi)=\lambda \phi^{2}$ is a better choice in studying the phantom divide crossing cosmology in the context of nonminimal scalar tensor teleparallel dark energy.

\section{Acknowledgments}

This work was supported by Akdeniz University, Scientific Research Projects Unit. 
[1] A.G. Riess et al., Astrophys. J. 116, 1009-1038 (1998)

[2] S. Perlmutter et al., Astrophys. J. 517 565-586 (1999)

[3] D.N. Spergel et al., Astrophys. J. Suppl. 148, 213 (2003)

[4] C.B. Netterfield et al., Astrophys. J., 571, 664 (2002)

[5] C.N. Bennett et al., Astrophys. J. Suppl. 148 1, (2003)

[6] M. Tegmark et al., Phys. Rev. D 69103501 (2004).

[7] E. J. Copeland,M. Sami, and S. Tsujikawa, Int. J. Mod. Phys. D. 15, 1753 (2006)

[8] R. Durrer, R. Maartens, Gen. Rel. Grav. 40, 301 (2008).

[9] B. Ratra, P.J.E. Peebles, Phys. Rev. D 37, 3406 (1988)

[10] C. Wetterich, Nucl. Phys. B 302, 668 (1988)

[11] A.R. Liddle, R.J. Scherrer, Phys. Rev. D 59, 023509 (1999)

[12] I. Zlatev, L.M. Wang, P.J. Steinhardt, Phys. Rev. Lett. 82, 896 (1999)

[13] R.R. Caldwell, Phys. Lett. B 545, 23 (2002)

[14] R.R. Caldwell, M. Kamionkowski, N.N. Weinberg, Phys. Rev. Lett. 91, 071301 (2003)

[15] S. Nojiri, S.D. Odintsov, Phys. Lett. B 562, 147 (2003)

[16] V.K. Onemli, R.P. Woodard, Phys. Rev. D 70, 107301 (2004)

[17] M.Z. Li, B. Feng, X.M. Zhang, J. Cosmol. Astropart. Phys. 512, 002 (2005)

[18] B. Feng, X.L. Wang, X.M. Zhang, Phys. Lett. B 607, 35 (2005)

[19] Z.K. Guo et al., Phys. Lett. B 608, 177 (2005)

[20] T.P. Sotiriou, V. Faraoni, Rev. Mod. Phys. 82, 451 (2010)

[21] A. De Felice, S. Tsujikawa, Living Rev. Relativ. 13, 3 (2010)

[22] S. Nojiri, S.D. Odintsov, Phys. Rep. 505, 59 (2011)

[23] S. Capozziello, M. De Laurentis, Phys. Rep. 509, 167 (2011)

[24] A. Einstein, Sitz.ber. Preuss. Akad. Wiss. Phys. Math. Kl. 217, (1928)

[25] A. Einstein, Sitz.ber. Preuss. Akad. Wiss. Phys. Math. Kl. 224, (1928)

[26] K. Hayashi and T. Shirafuji, Phys. Rev. D 19, 3524 (1979)

[27] G. R. Bengochea, R. Ferraro, Phys. Rev. D 79, 124019 (2009)

[28] E. Linder, Phys. Rev. D 81, 127301 (2010)

[29] P. Wu, H.W. Yu, Phys. Lett. B 693, 415-420 (2010)

[30] R. Myrzakulov, Eur. Phys. J. C 71, 1752 (2011)

[31] R. Zheng, Q.-G. Huang, J. Cosmol. Astropart. Phys. 03, 002 (2011)

[32] K. Bamba, C.-Q. Geng, C.-C. Lee, L.-W. Luo, JCAP, 01, 021 (2011)

[33] T. Wang, Phys. Rev. D 84, 024042 (2011)

[34] R.-J. Yang, Europhys. Lett. 93, 60001 (2011)

[35] G.R. Bengochea, Phys. Lett. B 695, 405 (2011)

[36] P. Wu, H.W. Yu,, Phys. Lett. B 692, 176 (2010)

[37] B. Li, T.P. Sotiriou, J.D. Barrow, Phys. Rev. D 83, 064035 (2011)

[38] Y. Zhang, H. Li, Y. Gong, Z.-H. Zhu, J. Cosmol. Astropart. Phys. 07, 015 (2011)

[39] C. Deliduman, B. Yapiskan, Absence of relativistic stars in $\mathrm{f}(\mathrm{T})$ gravity, arXiv:1103.2225

[40] S. Chattopadhyay, U. Debnath, Int. J. Mod. Phys. D 20, 1135 (2011)

[41] M. Sharif, S. Rani, Mod. Phys. Lett. A 26, 1657 (2011)

[42] S. Capozziello, V. Cardone, H. Farajollahi, A. Ravanpak, Phys. Rev. D 84, 043527 (2011)

[43] R. Ferraro, F. Fiorini, Phys. Rev. D 84, 083518 (2011)

[44] M.H. Daouda, M.E. Rodrigues, M. Houndjo, Eur. Phys. J. C 72, 1890 (2012)

[45] H. Farajollahi, A. Ravanpak, P. Wu, Astrophys. Space Sci. 338, 23 (2012)

[46] M. Jamil, D. Momeni, N. Serikbayev, R. Myrzakulov, Astrophys. Space Sci. 339, 37 (2012)

[47] K. Karami and A. Abdolmaleki, J. Cosmol. Astropart. Phys. 04, 007 (2012)J

[48] M. Jamil, D. Momeni, R. Myrzakulov, Eur. Phys. J. C 72, 2122 (2012)

[49] C.-Q. Geng, C.-C. Lee, E.N. Saridakis, Y.-P. Wu, Phys. Lett. B 704, 384 (2011)

[50] C.-Q. Geng, C.-C. Lee, E.N. Saridakis, J. Cosmol. Astropart. Phys. 01, 002 (2012)

[51] H. Wei, Phys. Lett. B 712, 430 (2012)

[52] C. Xu, E. N. Saridakis, G. Leond, J. Cosmol. Astropart. Phys. 07, 005 (2012)

[53] A. Banijamali, B. Fazlpour, Astrophys. Space Sci. 342,229235 (2012)

[54] S. Capozziello and R. de Ritis, Class. Quantum. Grav. 11,107 (1994)

[55] R. Ferraro, F. Fiorini, Phys. Rev. D 75, 084031 (2007)

[56] R. de Ritis, G. Marmo, G. Platania, C. Rubano, P. Scudellaro, C. Stornaiolo, Phys. Rev. D 42, 1091 (1990)

[57] M. Demianski, R. de Ritis, C. Rubano, P. Scudellaro, Phys. Rev. D 46, 1391 (1992)

[58] S. Capozziello, R. de Ritis, Phys. Lett. A 177, 1 (1993)

[59] S. Capozziello, Lambiase, Gen. Relativ. Gravit. 32, 673 (2000)

[60] A.K. Sanyal, B. Modak, Class. Quantum Gravit. 18, 3767 (2001)

[61] A.K. Sanyal, Phys. Lett. B 524, 177 (2002) 
[62] S. Kamilya, B. Modak, S. Biswas, Gen. Relativ. Gravit. 36, 661 (2004)

[63] U. Camci, Y. Kucukakca, Phys. Rev. D, 76, 084023 (2007)

[64] Y. Kucukakca, U. Camci, I. Semiz, Gen. Relativ. Gravit. 44, 1893 (2012)

[65] S. Capozziello, A. de Felice, J. Cosmol. Astropart. Phys. 08, 016 (2008)

[66] B. Vakili, Phys. Lett. B 664, 016 (2008)

[67] M. Roshan, F. Shojai, Phys. Lett. B 668, 238 (2008)

[68] A. Paliathanasis, M. Tsamparlis, S. Basilakos, Phys. Rev. D 84, 123514 (2011)

[69] I. Hussain, M. Jamil, F.M. Mahomed, Astrophys. Space Sci. 337, 373 (2012)

[70] Y. Kucukakca, U. Camci, Astrophys. Space Sci. 338, 211 (2012)

[71] M.F. Shamir, A. Jhangeer, A.A. Bhatti, Chin. Phys. Lett. 29, 080402 (2012)

[72] S. Capozziello, A. Stabile, A. Troisi, Class. Quantum Grav. 24, 2153 (2007)

[73] H. Wei, X. J. Guo, L. F. Wang, Phys. Lett. B 707, 298 (2012)

[74] K. Atazadeh, F. Darabi, Eur. Phys. J. C 72, 2016 (2012)

[75] M. Jamil, D. Momeni, R. Myrzakulov, Eur. Phys. J. C 72, 2137 (2012)

[76] H. Mohseni Sadjadi, Phys. Lett. B, 718, 270 (2012)

[77] C. Rubano and P. Scudellaro, Gen. Rel. Grav. 34, 307 (2002)

[78] M. Demianski, C. Rubano, and C. Tortora, Astron. Astrophys. 431, 27 (2005)

[79] S. Capozziello, R. de Ritis, C. Rubano, P. Scudellaro, Riv. Nuovo Cimento 19, 27 (1996)

[80] U. Alam, V. Sahni, A.A. Starobinsky, J. Cosmol. Astropart. Phys. 06, 008 (2004)

[81] S. Nesseris, L. Perivolaropoulos, J. Cosmol. Astropart. Phys. 01, 018 (2007)

[82] H.K. Jassal, J.S. Bagla, T. Padmanabhan, Mon. Not. Roy. Astron. Soc.P 405, 2639 (2010)

[83] J.S. Bagla, H.K .Jassal, T.Padmanabhan, Phys. Rev. D 67, 063504 (2003)

[84] J.M. Aguirregabiria, R. Lazkoz, Phys. Rev. D 69, 123502 (2004)

[85] E.J. Copeland, M.R. Garousi, M. Sami, S. Tsujikawa, Phys. Rev. D 71, 043003 (2005)

[86] G .Calcagni, A.R. Liddle, Phys. Rev. D 74, 043528 (2006) 